
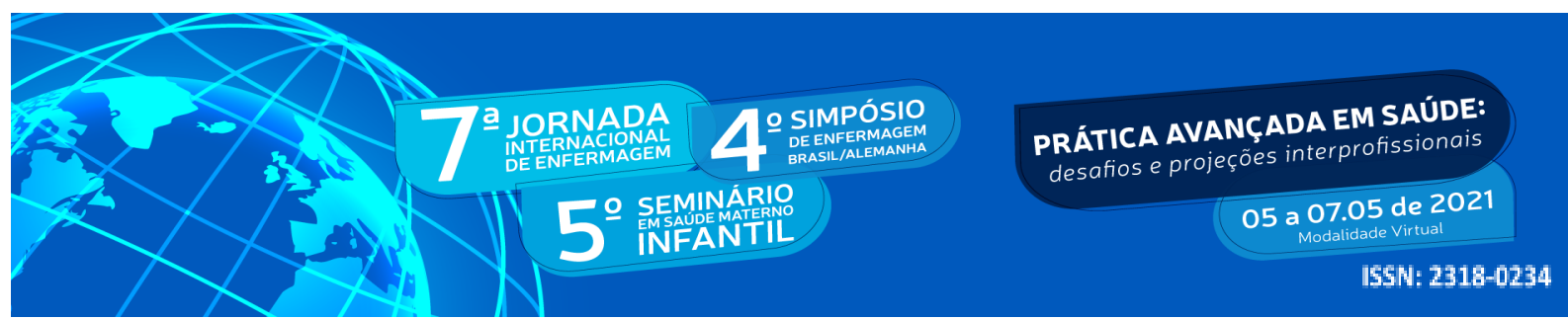

DOI: http://doi.org/10.48195/jie2021-186

\title{
SISTEMA ÚNICO DE SAÚDE: DA TEORIA À PRÁTICA ORGANIZACIONAL ${ }^{1}$
}

\author{
Andressa Caetano²; Tanise Pereira Santini ${ }^{3}$; Flavia Dorneles Saleh ${ }^{4}$; Maittê Vargas \\ Zago ${ }^{5}$; Marli Terezinha Stein Backes ${ }^{6}$; Dirce Stein Backes?.
}

\begin{abstract}
RESUMO
Objetivou-se conhecer a percepção de gestores de saúde sobre o Sistema Único de Saúde.. Método: Trata-se de um estudo exploratório descritivo, de abordagem qualitativa, realizado com 11 gestores de saúde da região central do Rio Grande do Sul. Os dados foram coletados entre os anos de 2017 e 2018, a partir de entrevistas individuais com questões norteadoras e analisadas pela técnica de análise de conteúdo temática. Resultados: Os dados codificados e interpretados resultaram em dois eixos temáticos: Abordagem tradicional de gestão em saúde e abordagem sistêmica de gestão e intervenção em saúde. Conclusão: Os gestores de saúde possuem conhecimento dos princípios e diretrizes que norteiam o sistema de saúde nacional, embora reproduzam um modelo de concepção e intervenção dicotômico, pontual e linear sustentado pelo modelo de gestão tradicional de pensar e agir em saúde.
\end{abstract}

Palavras-chave: Dinâmica não linear; Gestão em saúde; Sistema Único de Saúde.

\begin{abstract}
The objective was to get to know the perception of health professionals and managers about the Unified Health System. Method: This is a descriptive exploratory study, with a qualitative approach, carried out with 28 professionals and 14 health managers. Data were collected between March 2017 and October 2018, from individuals with guiding questions and related by thematic content analysis technique. Results: The coded and interpreted data resulted in two thematic axes: Traditional approach to health management and systemic approach to health management and intervention. Conclusion: Health professionals and managers have knowledge of the principles and guidelines that guide the

\footnotetext{
${ }^{1}$ Trabalho de Pesquisa - Fomentado por FAPERGS.

${ }^{2}$ Fisioterapeuta oncológica. Mestranda em Saúde Materno Infantil. Email: fisioterapiandressa@gmail.com

${ }^{3}$ Estudante do Curso de Enfermagem. Bolsista Probic/FAPERGS. Universidade Franciscana. E-mail: tanisesantini@hotmail.com

${ }^{4}$ Estudante do Curso de Enfermagem. Bolsista voluntária Probic/FAPERGS. Universidade Franciscana. E-mail: flaviasaleh222@gmail.com

${ }^{5}$ Estudante do Curso de Enfermagem. Bolsista voluntária Probic/FAPERGS. Universidade Franciscana. E-mail: maitte.zago@yahoo.com.br

${ }^{6}$ Enfermeira. Doutora em Enfermagem. Professora do Programa de Pós-Graduação de Enfermagem da

Universidade Federal de Santa Catarina. E-mail: marlibackes.ufsc@gmail.com

${ }^{7}$ Orientadora. Doutora em Enfermagem. Coordenadora do Mestrado em Saúde materno infantil. Universidade

Franciscana. E-mail: backesdirce@ufn.edu.br
} 


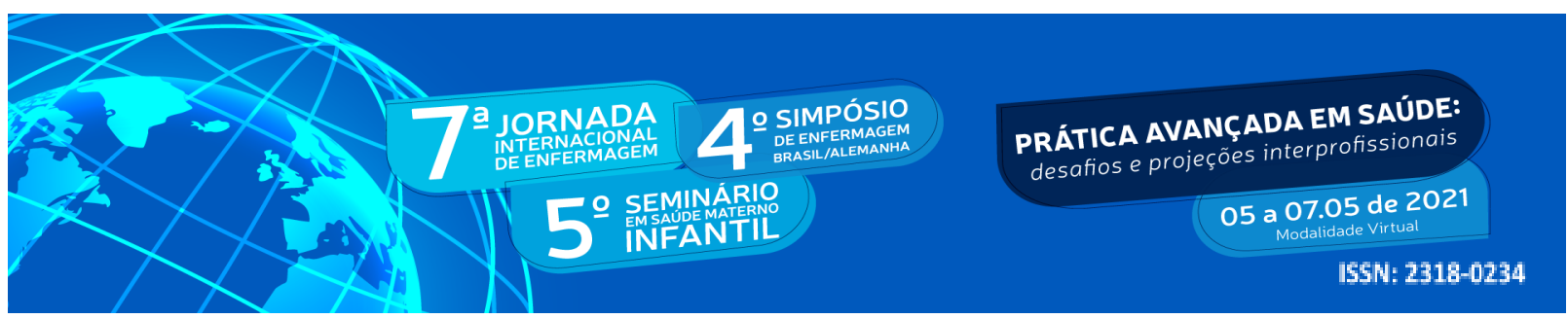

national health system, although they reproduce a dichotomous, punctual and linear design and intervention model supported by the traditional management model of thinking and acting in health.

Key Words: Nonlinear Dynamics; Health Management; Unified Health System.

\section{INTRODUÇÃO}

Com a institucionalização do Sistema Único de Saúde (SUS) se instaurou, no Brasil, um novo processo de gestão e intervenção em saúde. Transcende-se, teoricamente, de um enfoque tradicional clássico a uma abordagem sistêmica articulada em redes e linhas de cuidado em saúde. Segue a mesma doutrina e os mesmos princípios organizativos em todo o território nacional, sob a responsabilidade das três esferas autônomas governamentais. Orienta-se, com base na Constituição Federal de 1988, nos princípios como a integralidade, a universalidade e a equidade, descentralizando a gestão entre os vários entes federativos que possuem legislação e normas específicas (SOUTO; OLIVEIRA, 2016; VICTORA et al., 2011)

A abordagem clássica de gestão foi tradicionalmente influenciada por princípios ordenadores deterministas, como o reducionismo, o pensamento analítico e o mecanicismo dominantes em todas as ciências. O reducionismo se foca na crença de que os fenômenos podem ser reduzidos em entidades mais simples possíveis, o pensamento analítico decompõe o todo em partes mais simples para explicá-las e o mecanicismo, se conduz pela relação de causa e efeito entre os fenômenos (CHIAVENATO, 2009; DE SORDI, 2012; DRUCKER, 2012).

A abordagem sistêmica transcende, em contrapartida, ao paradigma da simplificação ao questionar os reducionismos provocados pela fragmentação e a mecanização dos fenômenos e ao se apoiar em princípios como: auto-organização, conectividade, imprevisibilidade, interdisciplinaridade, multidimensionalidade, dentre outros (MORIN, 2011). Para tanto, o referencial sistêmico oferece subsídios importantes à concepção integralizadora, universal e regionalizada do SUS, questionando os reducionismos e as fragmentações que tem pautado tanto as investigações científicas quanto às intervenções no âmbito da prevenção, promoção, proteção e reabilitação em saúde. 


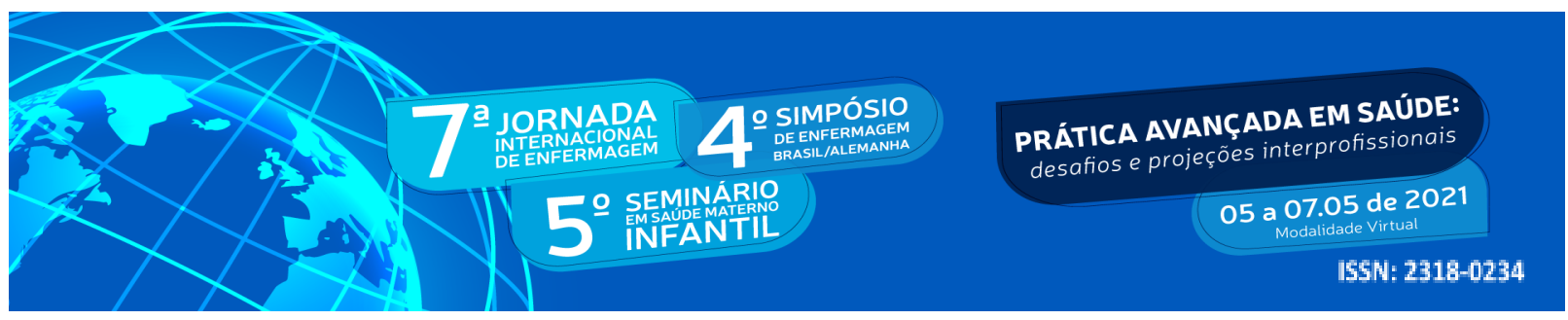

$\mathrm{Na}$ área da saúde vivencia-se ainda, um cenário fortemente determinado, pelo pensamento fragmentado, hiperespecializado e reprodutor de modelos assistenciais hegemônicos de intervenção. Este pensar reducionista, na visão de pesquisadores da área, é considerado um dos principais obstáculos para o alcance de resultados efetivos e eficazes, tanto no SUS quanto em outros sistemas de saúde mundiais (BOSSER; MITCHELL, 2011; BARRETO, 2017; ARRUDA et al., 2015; BACKES et al., 2012; BACKES et al., 2016).

\section{OBJETIVO}

Conhecer a percepção de gestores de saúde sobre o Sistema Único de Saúde.

\section{METODOLOGIA}

Trata-se de um estudo exploratório descritivo, de abordagem qualitativa, realizado com o propósito de construir novos conhecimentos e proporcionar novas perspectivas para a área da saúde (STRAUSS; CORBIN, 2008).

A coleta dos dados foi realizada por meio de entrevistas individuais com Gestores de Serviços de Saúde da região central do Rio Grande do Sul. Os participantes foram escolhidos de forma aleatória, a partir de um convite formal. As entrevistas foram realizadas em dias e horários previamente agendados entre os anos de 2017 e 2018 . Foram incluídos gestores com mais de dois anos de atuação na gestão e excluídos os gestores que não atuavam diretamente no Sistema Único de Saúde, de modo que a amostra foi composta por 11 gestores.

Duas questões básicas nortearam as entrevistas: Fale-me sobre o que você sabe e pensa do SUS. Como você percebe a dinâmica de trabalho no contexto do SUS?

Após a transcrição das entrevistas, o material empírico foi submetido à análise de conteúdo temática (BARDIN, 2016), a fim de descobrir os núcleos de sentido que compunham a comunicação, cuja presença ou frequência acrescentavam perspectivas significativas ao objeto de estudo. A noção da temática esteve associada às afirmações recorrentes apresentadas por palavras, frases ou ideias. Assim, a operacionalização do processo seguiu as três etapas preconizadas para a realização da análise de conteúdo temática. 


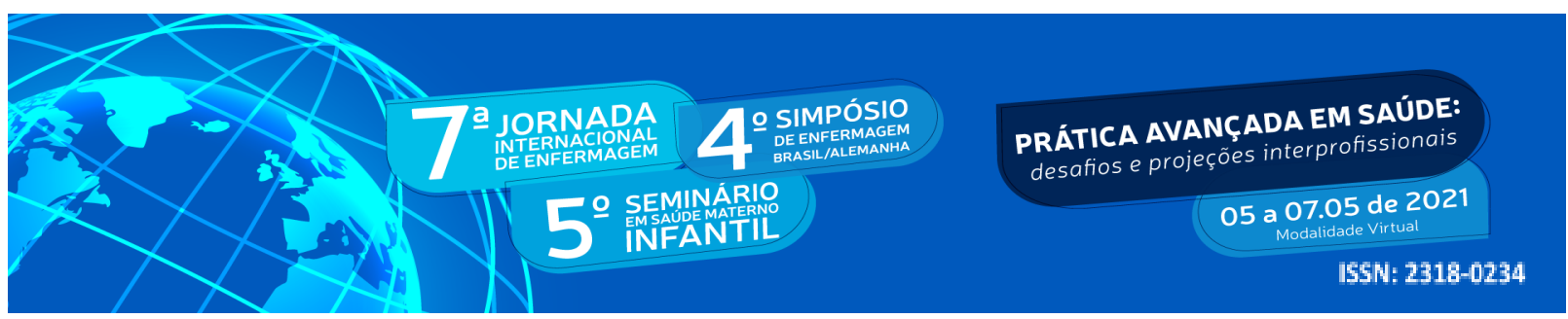

Na pré-análise foi realizada leitura exaustiva dos dados, com a organização do material e da formulação de hipóteses. A segunda etapa foi a exploração do material para codificação dos dados brutos. Na terceira etapa fez-se a interpretação dos dados e delimitados os eixos temáticos, a partir da compreensão dos significados estabelecidos (BARDIN, 2016).

Os critérios éticos atendem às recomendações da Resolução CNS n 466/2012 que prescreve a ética em pesquisa com seres humanos (BRASIL, 2012). O projeto foi submetido e aprovado pelo Comitê de Ética em Pesquisa sob o n. 4.253.905.

Para preservar o anonimato, os gestores foram identificados com a letra "G", seguida de um algarismo numérico, conforme segue (G1, G2, G3... G11).

\section{RESULTADOS E DISCUSSÃO}

A análise dos dados resultou em dois eixos temáticos: Abordagem tradicional de gestão em saúde e abordagem sistêmica de gestão e intervenção em saúde.

\section{Abordagem tradicional de gestão em saúde}

Ficou notória na percepção do G1 que, na maioria das vezes, precisam discorrer de um ponto para o outro da rede para conseguir a assistência necessária, conforme depoimento: Depois que você entra no sistema tudo funciona... o difícil é entrar, por que te mandam de um lugar para o outro e tudo demora muito. Destacaram que alguns profissionais, em muitos casos, desconhecem o fluxo orientador da rede e que não são claros e objetivos ao encaminhá-los aos serviços pertinentes. Desaprovam, as longas filas de espera, a retirada de fichas médicas na madrugada, desagendamento de consultas, exames e/ou cirurgias, e a desinformação em relação a determinadas condutas médicas, dentre outros percalços alienantes, nos quais se consideram meros objetos do sistema de saúde.

De acordo com um dos gestores, o SUS não funciona como deveria na prática. Apesar de não conseguirem imaginar o Brasil sem o SUS, sinalizam fragilidades no processo de trabalho, na forma de dinamizar e gerir o sistema. As fragilidades mencionadas foram: excessiva compartimentalização do trabalho, centralização hierárquica das decisões, rigidez nos padrões de controle, inflexibilidade das escalas de serviço, desvalorização profissional, os 


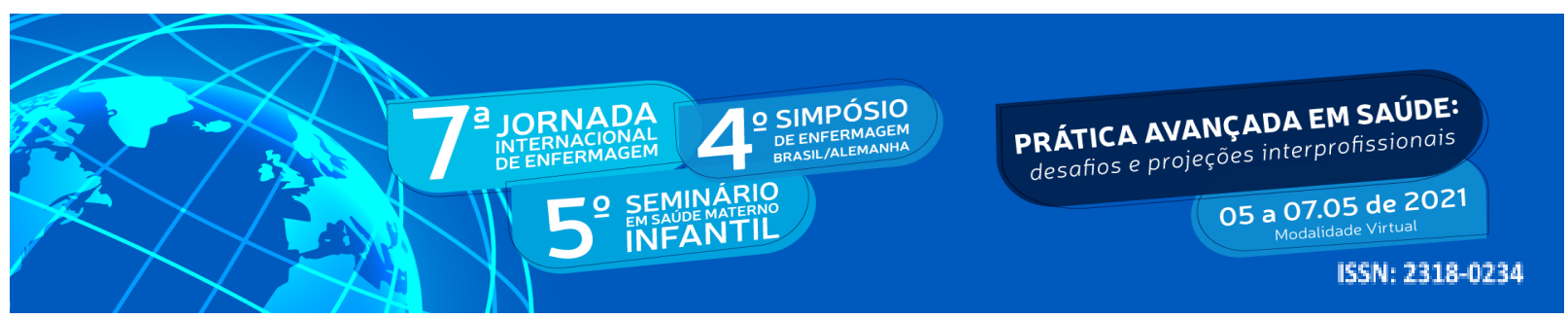

conflitos inter-relacionais entre as equipes. Conforme a fala: As pessoas conversam pouco... cada serviço tenta fazer a sua parte da melhor forma, mas poucos sabem conversar com o todo da rede $(\mathrm{G} 4)$.

O G7 destacou fragilidades na organização e na dinamização da rede de saúde municipal e regional. Que os serviços atuam de forma pouco interativa e cooperativa e que, nessa lógica verticalizada de trabalho, cada um busca assegurar o funcionamento de sua parte, sem, contudo, perceber a atenção integral da rede. Referiram que os serviços não conversam entre si e que os processos são conduzidos de forma fragmentada e na base da culpabilização de uns contra os outros. Foi apontada a falta de lideranças para a condução integral dos serviços, pouca participação e comprometimento dos colaboradores no alcance das metas institucionais. Alguns poucos gestores atribuíram os entraves do setor da saúde aos Órgãos superiores, aos quais são atribuídas a responsabilidade pela não efetivação dos princípios e diretrizes do SUS.

\section{Abordagem sistêmica de gestão e intervenção em saúde}

De modo geral, os gestores reconheceram que o SUS é um sistema único para todos os brasileiros, como também um sistema respeitado pela possibilidade do acesso universal a consultas, exames, medicamentos e cirurgias, além da visita dos agentes de saúde da família e outros. Retrataram uma concepção fortemente assistencialista e utilitarista do sistema com foco na doença. Tal concepção reflete, em parte, as práticas de educação e intervenção em saúde verticalizadas e lineares que têm vigorado neste e em outros setores.

Para o G2, o SUS é uma teia/rede perfeita e o melhor plano de saúde nacional, no qual muitos outros países se espelham. Reconhecem o alcance das diferentes classes econômicas, abrangendo desde os serviços básicos aos mais complexos, mas que apresentam dificuldades operacionais. Ao mesmo tempo em que percebem o sistema como precário e deficiente, reconhecem que este já faz parte da história e da vida dos brasileiros, constituindo em bem social. Reforçam que é uma rede perfeita, mas que na prática os fios/setores não conversam entre si nem potencializam soluções coletivas. 


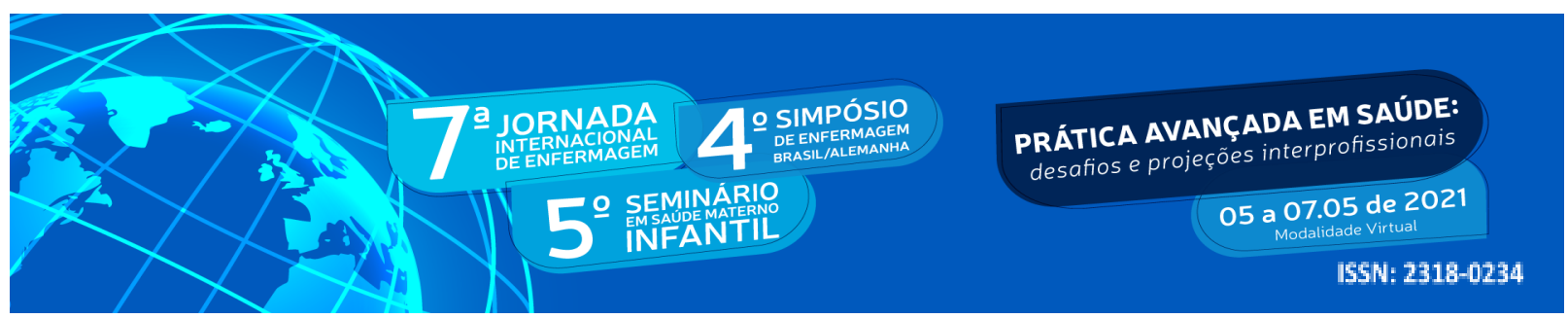

De modo geral, os gestores têm conhecimento dos princípios e das diretrizes que regem o SUS e reforçam a importância do mesmo para a saúde ampliada dos brasileiros. Um dos gestores, em especial, conseguiu ampliar a concepção em relação aos princípios e diretrizes que norteiam o SUS, enfatizando que é um sistema completo e/ou um sistema guarda-chuva norteando a saúde dos brasileiros em suas diferentes dimensões e complexidades. Sustentam que o SUS se dinamiza como rede, na qual diferentes pontos se conectam, compartilham e se complementam, embora, por vezes, os fios se apresentem frágeis e descontínuos.

Foi mencionado pelo G6 que, em contrapartida, convive-se com uma cultura social de que o SUS é bom enquanto recebo as coisas de graça. Enfatizaram que a população está culturalmente habituada ao imediatismo e à gratuidade. Mas o SUS é um sistema completo e precisa atuar em rede e centrar-se, prioritariamente, no fortalecimento da atenção básica de saúde.

O G11 reconhece, mesmo que de modo superficial, os princípios e as diretrizes que norteiam o SUS e que requer a organização e a dinamização dos serviços em redes e linhas de cuidado, nas quais os pontos conectivos se constituem em espaços dialógicos para o efetivo funcionamento do sistema como um todo.

O SUS é, por si só, um sistema complexo. Logo, tanto mais complexo será discuti-lo, no sentido de delinear possibilidades teórico-práticas que contribuam para a sua dinamização e operacionalização sistêmica, enquanto sistema único e articulado em redes e linhas de cuidado, na prática. Percebe-se que não basta a garantia constitucional de um sistema único, regionalizado e completo. Mais do que isto é preciso ampliar e instrumentalizar os autores e atores do SUS com referenciais que sejam capazes de transcender a linearidade do ser, pensar, falar e agir em saúde, bem como reordenar o modelo assistencial vigente e hegemônico no país.

Ao iniciar este processo de discussão emergiram, imediatamente, os seguintes questionamentos: Por que o SUS é considerado um sistema completo, concebido e articulado em redes, mas que não consegue ser operacionalizado de forma efetiva e resolutiva na prática? $\mathrm{O}$ que está na raiz dos modelos ditos tradicionais e/ou piramidais de gestão em saúde 


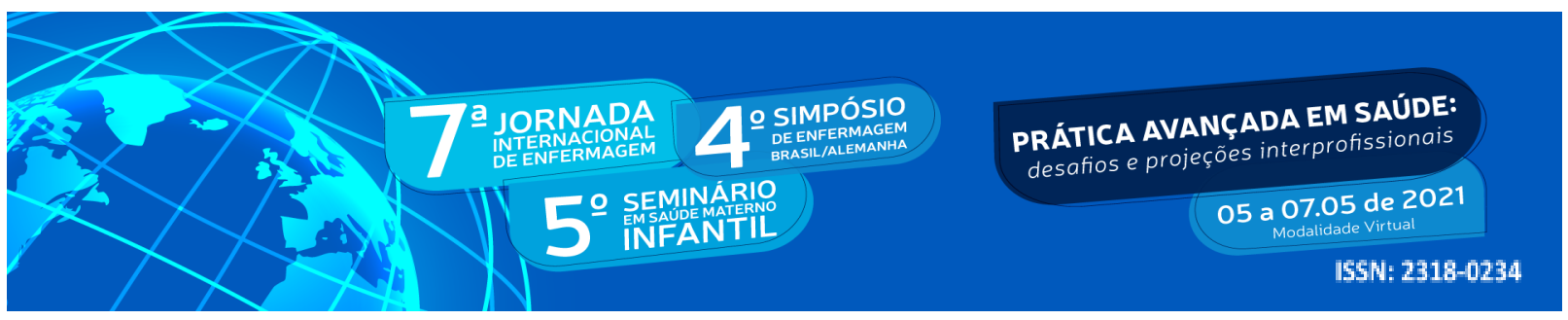

capazes de perpetuar intervenções verticalizadas e reducionistas? Quais estratégias teórico-práticas são necessárias para efetivar, concretamente, o SUS como sistema dinâmico, integralizado e articulado em redes?

A abordagem sistêmica surgiu, portanto, como antítese à abordagem tradicional mecanicista, na qual os processos de condução das organizações eram verticalizados, fragmentados e deterministas. Surgiu da necessidade de se pensar não mais em máquinas com funcionalidade linear e fechada, mas em sistemas ou em organizações orgânicas e mutáveis. Caracteriza-se sistema, neste caso o sistema SUS, como unidade complexa na qual se movem elementos interdependentes e interligados para formar o todo complexo, aqui traduzido pelos fios que se entrecruzam para formar a rede integral de atenção à saúde (MORIN, 2011).

Numa organização sistêmica as pessoas, os serviços, as funções e a gestão são interdependentes e componentes de um sistema que é a própria organização. Qualquer mudança numa das partes e/ou nós da rede, afeta simultaneamente o sistema como um todo. Sob esse pensar, o sucesso e/ou insucesso de um sistema é determinado pela qualidade das interações dos serviços e o sistema, o sistema e outros sistemas e o sistema e seu ambiente total (CHIAVENATO, 2009).

Nessa condição, como qualificar as relações e as interações entre os serviços e organizações que formam o SUS? Como dinamizar a rede integral de saúde pela participação e o engajamento efetivo de todos os atores envolvidos na dinamização do SUS? Como demonstrar que além dos gestores e profissionais de saúde os usuários se constituem em elementos agregadores e indutores de uma nova ordem sistêmica?

O SUS é um sistema único porque segue a mesma doutrina e os mesmos princípios organizativos em todo o território nacional, sob a responsabilidade das três esferas autônomas de governo: federal, estadual e municipal. Pelo princípio da Universalidade, o SUS garante o direito de acesso a todos os serviços de saúde a todo e qualquer cidadão em âmbito nacional. Com o princípio da equidade, o SUS assegura ações e serviços de acordo com os níveis de complexidade de seus usuários. E através da integralidade, é assegurada a garantia de que cada pessoa é um todo indivisível e integrante de um sistema maior (ALBRECHT; ROSA; BORDIN, 2017). 


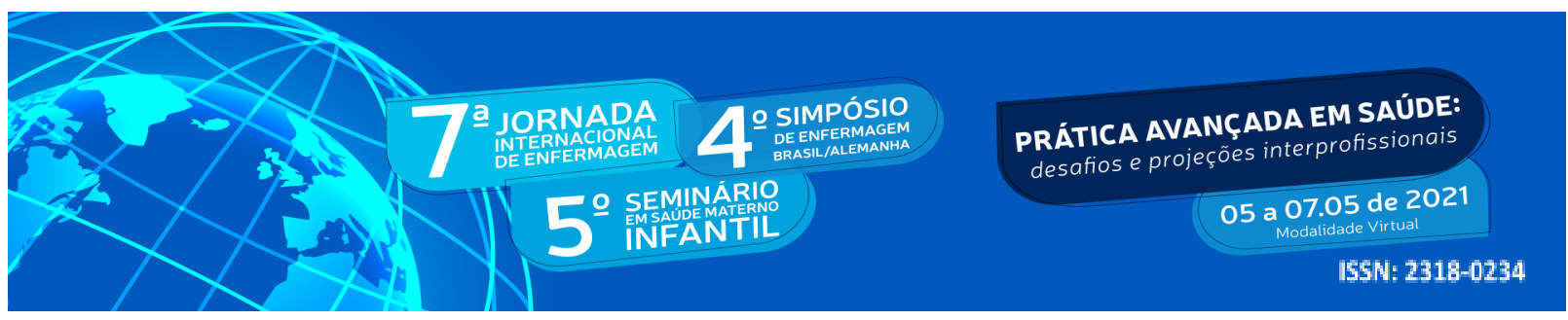

Concorda-se com pensadores da área da saúde onde enfatizam que para a construção do SUS necessário e desejado é imprescindível a reorientação do modelo assistencial vigente e hegemônico no país. Observa-se, que o modelo assistencial é uma das áreas nas quais se concentram os maiores entraves de um sistema de saúde. Dentre os princípios e as diretrizes do SUS, o da integralidade confronta incisivamente as práticas hegemônicas, tais como o reducionismo e a fragmentação, a objetivação dos sujeitos e o enfoque na doença e na intervenção pontual e assistencialista (ARRUDA et al., 2015; VARGAS et al., 2014).

Com base na abordagem sistêmica do SUS, os gestores devem se concentrar cada vez menos em direcionar e controlar os processos internos e mais em desenvolver as potencialidades e as iniciativas dos colaboradores. Os gestores exercem, portanto, papel fundamental de criação e renovação às condições mutáveis do ambiente, pela compreensão do todo nas partes do sistema e vice-versa, pela capacidade de dialogar e conviver com as diferenças, negociar em situações novas e de incertezas e integrar as noções de ordem e desordem. Além disso, se requer do gestor sensibilidade para perceber, compreender e lidar com ruídos e perturbações advindas do ambiente, no sentido de receber e fornecer interações qualificadas para a dinamização e consolidação das redes de atenção à saúde (BACKES et al., 2014).

A sociedade atual e do futuro é e será essencialmente dinâmica e mutável. Para acompanhar o ambiente turbulento e mutável, as organizações precisam ser orgânicas, isto é, dinâmicas, inovadoras e motivadas de forma permanente pelas interações e associações sistêmicas (CHIAVENATO, 2009).

\section{CONCLUSÃO}

Os profissionais e gestores de saúde possuem conhecimento dos princípios e diretrizes que norteiam o sistema de saúde nacional, embora reproduzam um modelo de concepção e intervenção dicotômico, pontual e linear sustentado pelo modelo de gestão tradicional de pensar e agir em saúde.

A percepção dos gestores sobre o SUS demonstra que não basta institucionalizar um sistema único de saúde para se obter resultados efetivos e eficazes. Além de uma concepção 


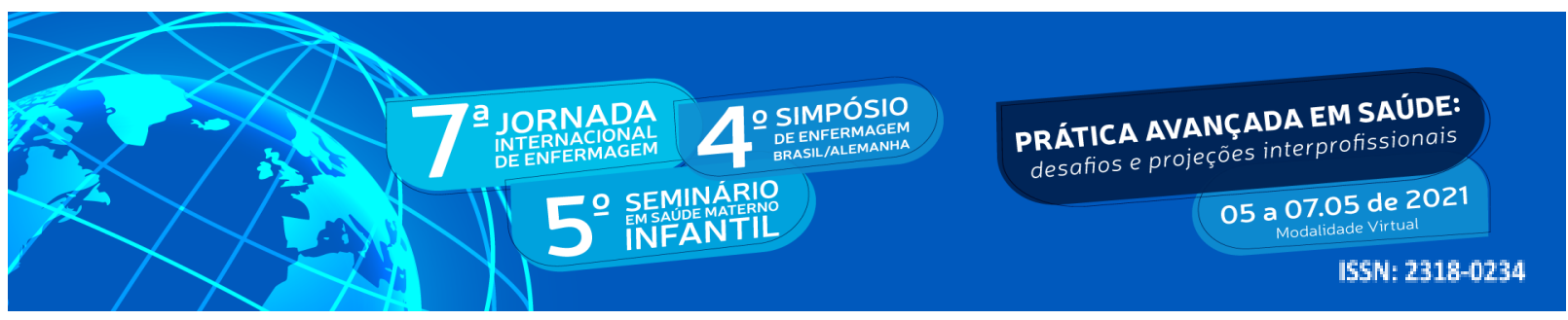

teórico-sistêmica do SUS é preciso que este referencial - sistêmico - encontre significado no pensar, na comunicação interativa e no agir profissional, a partir de um processo de auto-organização e qualificação das relações e interações entre as partes e das partes com o todo e vice-versa.

Nessa dinâmica auto-organizativa do SUS, os gestores assumem uma função cada vez mais proativa e integralizadora no que se refere à identificação das necessidades de cuidado da população, bem como no delineamento de políticas emancipatórias que promovam a qualificação das relações e interações do cuidado em saúde e o reordenamento do modelo de gestão e intervenção.

Com base na concepção teórico-sistêmica do SUS, já reconhecida e firmada como modelo ordenador e dinamizador do sistema, como transcender da teoria para a prática da gestão integrada e articulada em redes e parcerias?

\section{REFERÊNCIAS}

Albrecht, C.A.M.; Rosa, R.S.; Bordin, R. O conceito de equidade na produção científica em saúde: uma revisão. Saúde Soc. São Paulo, v.26, n.1, p.115-128, 2017.

Arruda, C. et al. Redes de atenção à saúde sob a luz da teoria da complexidade. Esc. Anna Nery, v.19, n.1, p.169-73, 2015.

Backes, D.S. et al. Systemic Interactivity between Interdependent Concepts of Nursing Care. Aquichán, v.16, n.1, p.24-31, 2016.

Backes, D.S. et al. The Idealized Brazilian Health System versus the real one: contributions from the nursing field. Rev. Latino-Am. Enfermagem, v.22, n.6, p.1026-33, 2014.

Backes, D.S. et al. The role of the nurse in the Brazilian Unified Heath System: from community health to the family health strategy. Ciênc. saúde coletiva, v.17, n.1, p.223-30, 2012.

Bardin, L. Análise de conteúdo. Lisboa: Edições 70; 2016.

Barreto, M.L. Desigualdades em Saúde: uma perspectiva global. Ciência \& Saúde Coletiva, v.22, n.7, p.2097-2108, 2017. 


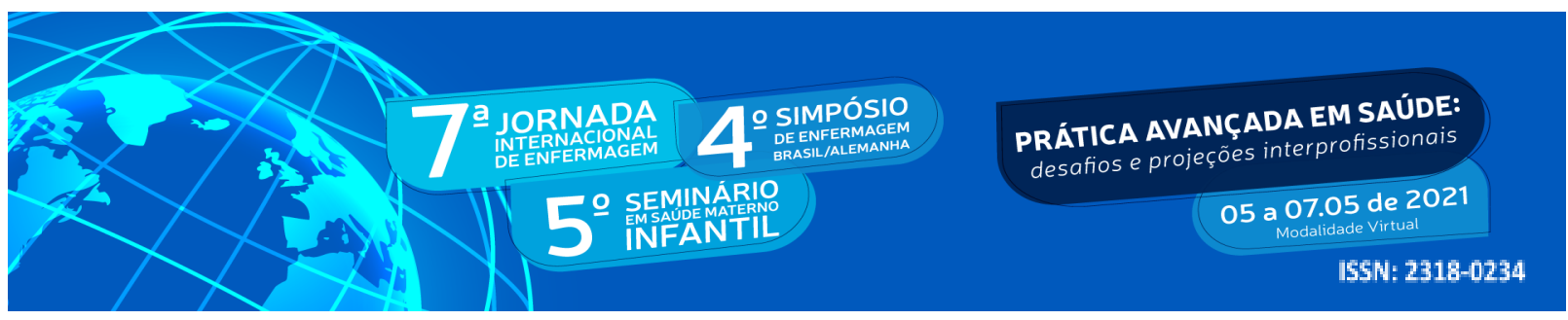

Bossert, T.J.; Mitchell, A.D. Health sector decentralization and local decision-making: decision space, institutional capacities and accountability in Pakistan. Social Science and Medicine, v.72, n.4, p39-48, 2011.

Brasil. Ministério da Saúde. Conselho Nacional de Saúde. Diretrizes e normas regulamentadoras de pesquisa envolvendo seres humanos. Resolução n. 466, de 12 de dezembro de 2012. Brasília: Ministério da Saúde; 2012

Chiavenato, I. Introdução à teoria geral de administração. $7^{\mathrm{a}}$ ed. Rio de Janeiro: Campus; 2009.

De Sordi, J.O. Gestão por processos: uma abordagem da moderna administração. São Paulo: Saraiva; 2012.

Drucker, P.F. Tecnologia, administração e sociedade. Rio de Janeiro: Elsevier; 2012.

Morin, E. Introdução ao pensamento complexo. 4ª ed. Porto Alegre (RS): Sulina; 2011.

Souto, L.R.; Oliveira, M.H.B. Movimento da Reforma Sanitária Brasileira: um projeto civilizatório de globalização alternativa e construção de um pensamento pós-abissal. Saúde debate, Rio de Janeiro, v. 40, n. 108, p. 204-218, 2016.

Strauss, A.; Corbin, J. Pesquisa qualitativa: técnicas e procedimentos para o desenvolvimento da teoria fundamentada. Porto Alegre, Artmed, 2008.

Vargas, I. et al. Regional-based Integrated Healthcare Network policy in Brazil: from formulation to practice. Health Policy Plan, v.23, n.2, p.1-13, 2014.

Victora, C.G. et al. Health conditions and health-policy innovations in Brazil: the way forward. Lancet, v.377, n.9782, p.2042-53, 2011. 